\title{
Application of the relocation-error distribution on geomagnetic databases. Analyses on the «Historical Italian Geomagnetic Data Catalogue»
}

\author{
Lluís Casas $\left({ }^{1}\right)$ and Alberto Incoronato $\left({ }^{2}\right)$ \\ (1) Departament de Geologia, Universitat Autònoma de Barcelona, Spain \\ (2) Dipartimento di Scienze della Terra, Università degli Studi di Napoli, Italy
}

\begin{abstract}
The reliability of the Historical Italian Geomagnetic Data Catalogue, comprising 536 directions and 393 intensities, has been assessed by comparing the historical geomagnetic measurements with the GUFM1 model predictions. Such measurements were assessed at three selected relocation centres. For all the data contained in the Catalogue it has been calculated the discrepancy between the relocated data and the GUFM1-model prediction at the relocation centres. There is a correlation between relocation distance and the mean discrepancy. The upper limit of discrepancy assumable as relocation error has been selected using error distributions previously calculated using geomagnetic field models. Angular and intensity threshold lines have been slightly shifted upwards to account for the estimated error of GUFM1 model itself at the considered region, mainly due to the crustal field. The Italian database proved to contain reliable data, as only a very low percentage of data (namely 14 directions and 20 intensities) can be considered anomalous. Possible explanations for such questionable data are suggested. All the remaining data of this catalogue could thus be added to the databases used to produce regional or global geomagnetic models.
\end{abstract}

Key words geomagnetism - magnetic dating - relocation

\section{Introduction}

The geomagnetic field is commonly described through its potential, which can be represented by a spherical harmonic series. A series of coefficients (that are referred to as Gauss coefficients) of the spherical functions are enough to accurately depict the features of the field for a given time. The International Associ-

Mailing address: Dr. Lluís Casas, Departament de Geologia, Universitat Autònoma de Barcelona, Edifici C, Campus de la UAB, 08193 Bellaterra (Cerdanyola del Vallès), Spain; e-mail: lluis.casas@uab.cat ation of Geomagnetism and Aeronomy (IAGA) publishes regularly estimates of the Gauss coefficients at five-yearly intervals (Maus et al., 2005) these constitute the so-called IGRF models that cover the evolution of the geomagnetic field from 1900 up to present. Another set of time-dependent Gauss coefficients, covering the interval from 1590 to 1990 , compose the GUFM1 model by Jackson et al. (2000). The historical part of the model is essentially based on ship log books.

Recently, both geomagnetic models (IGRF9 and GUFM1) have been used to compute the distribution of errors due to relocation of geomagnetic data (Casas and Incoronato, 2007). Relocation is routinely carried out by archaeomagnetists and consists on reducing data to a central location. This procedure permits to compare geomagnetic data from different loca- 
tions. The relocation process is usually performed using the «conversion via pole» method (CVP) and assumes a purely dipolar field within the area where the relocation is done. As is stated in Casas and Incoronato (2007) the distribution of relocation errors can be used by archaeomagnetists as an indication of the maxi- mum error induced by such a practice. However, this conclusion is restricted to periods with similar or lower values of the ratio between dipole to non-dipole contributions than in the present geomagnetic field; this could cover at least the last 7000 years (Korte and Constable, 2005).

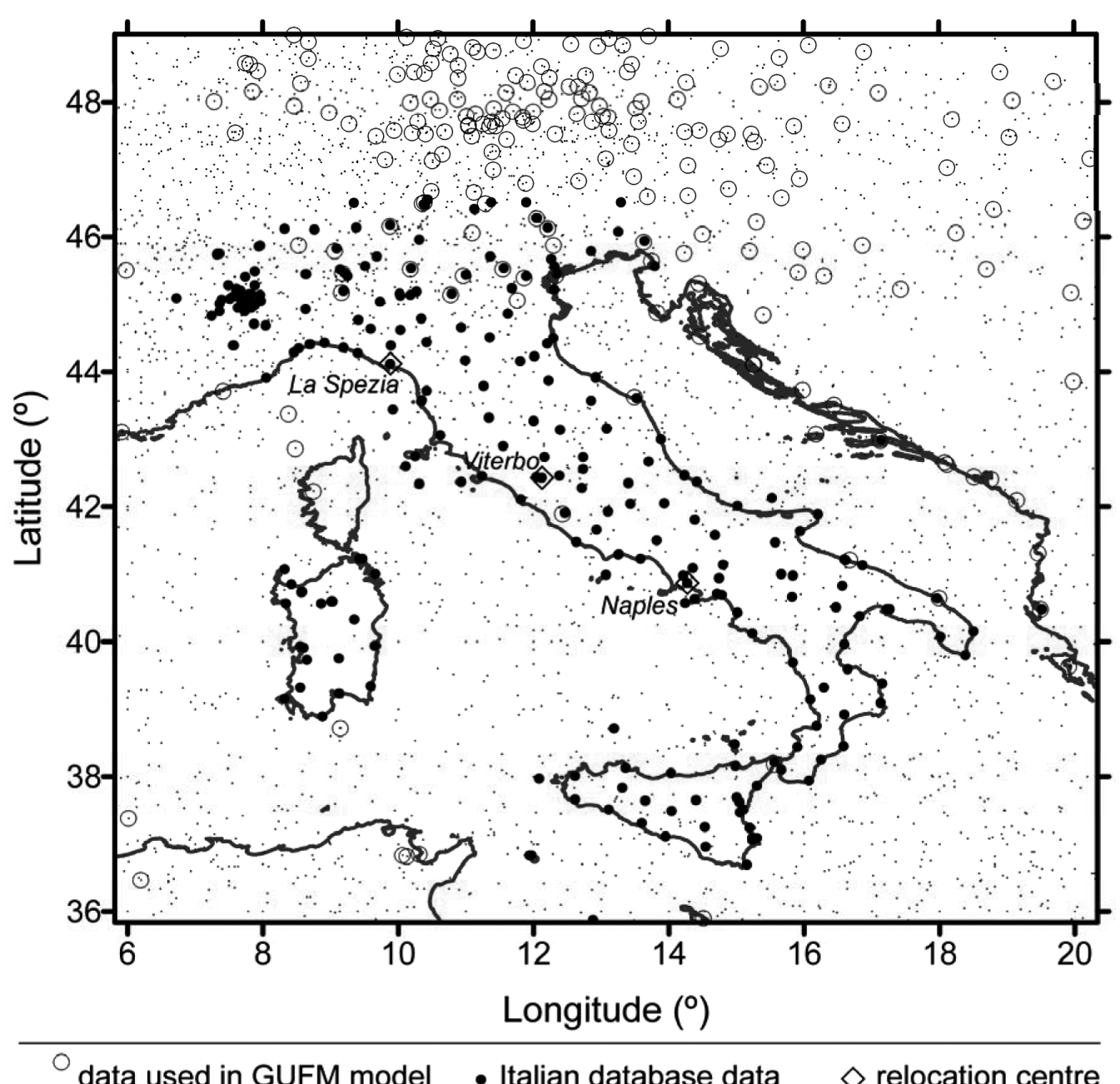

Fig. 1. Map of Italy and neighbouring areas with indication of several data locations: solid dots $(\bullet)$ indicate declination and inclination data at a given time from the Historical Italian Geomagnetic Data Catalogue (Cafarella et al., 1992a); open dots $(\bigcirc)$ indicate declination and inclination data at a given time from the database used to compute GUFM1 model; small dots (.) indicate single data values (declination or inclination) from the database used to compute GUFM1 model and finally open diamonds $(\diamond)$ indicate the three locations selected as relocation centres. 
Another possible application of the distribution of relocation errors can be found on observatory data from historical periods (last 400 years). The error distribution can be used to detect spurious data within historical geomagnetic databases. The historical Italian geomagnetic data catalogue (Cafarella et al., 1992a) has been used as a demonstrative example. This database, fully available at the cited reference (Cafarella et al., 1992a) is an excellent compilation of declination, inclination and intensity data (both isolate and simultaneous determinations essentially from the $19^{\text {th }}$ century) from many locations in Italy obtained from libraries of old institutes and universities, as well as data from Italian observatories (Cafarella et al., 1992b).

The limited resolution of historical (and older) models that often results in an imperfect representation of the non-dipole field structure, brings a worthy motive to apply the relocationerror distribution, which checks the self-consistency of data assemblies, instead of founding decisions on data quality solely on a geomagnetic field model. Moreover, the methodology used and presented in this paper allows a quick survey on long lists of geomagnetic data without the need of computing the «true» values (from a global model) at every single location but only at a few selected relocation centres.

\section{Analysis on the Historical Italian Geomagnetic Data Catalogue}

\subsection{Methodology}

The geomagnetic field for the time period of the data from the Italian catalogue is well described by the GUFM1 model (Jackson et al.,

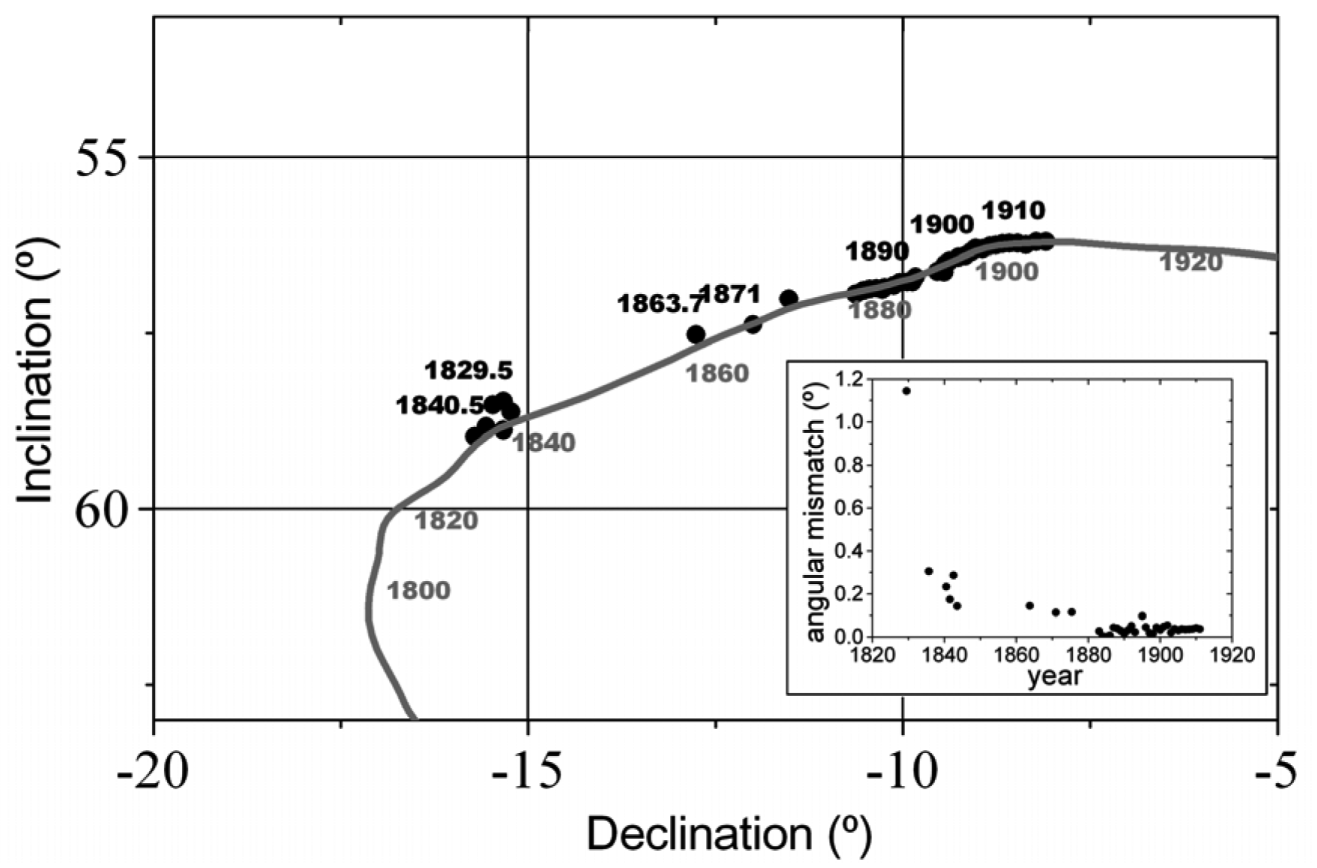

Fig. 2. Comparison between the Neapolitan geomagnetic data, circles, from the Historical Italian Geomagnetic Data Catalogue (Cafarella et al., 1992a) and the GUFM1 (Jackson et al., 2000) field model, grey line, in Naples. Inset: angular mismatch between data from the Catalogue and the corresponding values computed using GUFM1 model. 


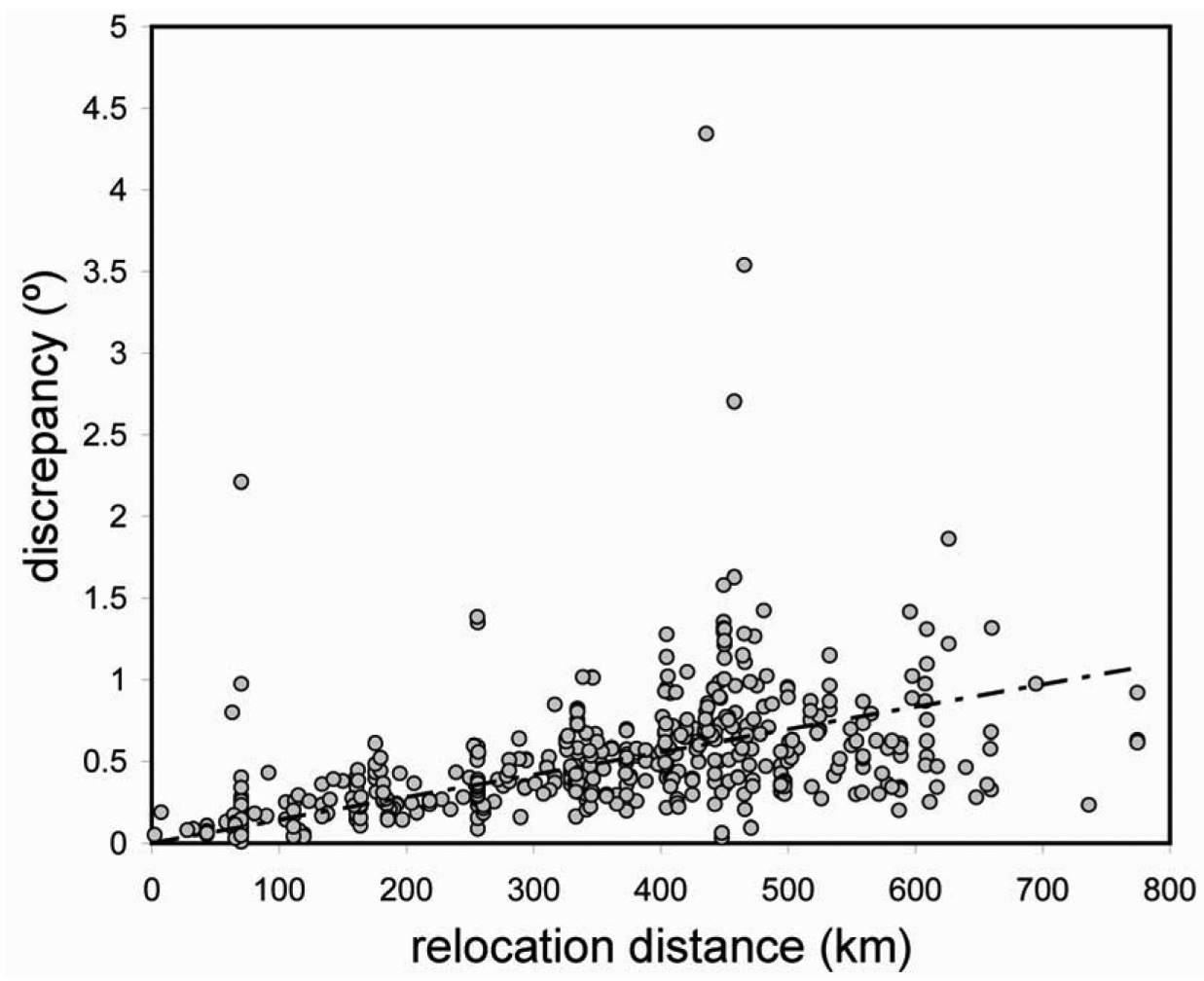

Fig. 3. Angular discrepancy between the relocated Italian-catalogue data and GUFM1 values at Viterbo as a function of relocation distance. Correlation is apparent.

2000). It is worth to note that data from the Italian catalogue were not used to build the GUFM1 model (see fig. 1), one of the authors of this model (A. Jackson) kindly provided us with the entire database used in his model and he also confirmed in a personal communication with the authors that the Italian catalogue was not used to develop GUFM1. However, the consistency between the Italian catalogue and the model can be assessed by comparing the historical geomagnetic measurements made in Naples (which is the most represented location within the Italian catalogue) with the model predictions for this location (fig. 2), coefficient $\mathrm{R}^{2}$ is 0.995 .

The calculation of the relocation error for any given geomagnetic datum requires the knowledge of the «true» value of the magnitude considered (direction or intensity) at the reloca- tion centre. Relocation error distributions presented in Casas and Incoronato (2007) were calculated considering a grid of relocation centres. For each of them, geomagnetic data from neighbouring sites (within a radius of $1200 \mathrm{~km}$ ) obtained from a model (IGRF or GUFM1) were relocated. The relocation error was calculated as the difference between the relocated data and the data predicted by the model at the relocation centre. Relocation error distributions calculated using the historical database were computed similarly. The «true» values at the relocation centres were calculated using the GUFM1 geomagnetic field model.

Data containing both declination and inclination of a given site and time were selected from the Italian catalogue to carry out the directional analysis. For the intensity analysis, data containing both inclination and the horizontal 


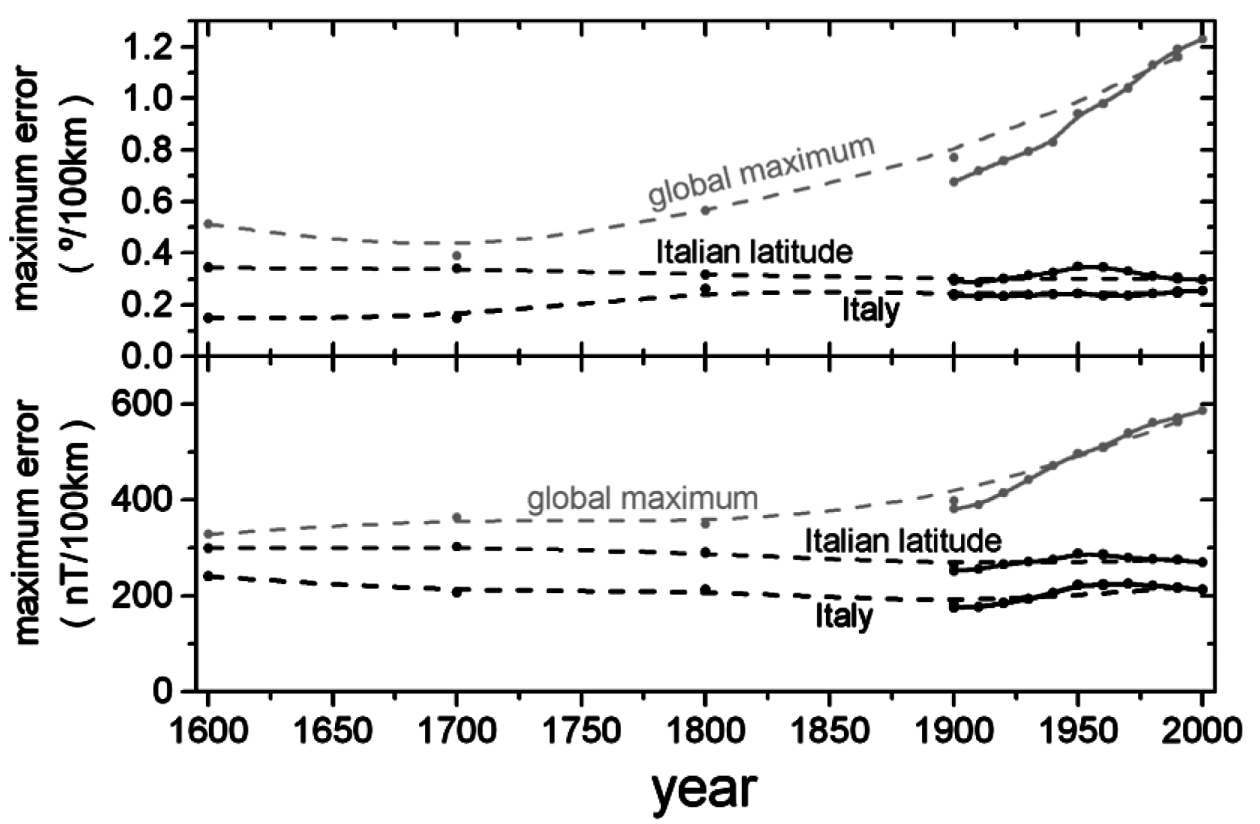

Fig. 4. Evolution of the maximum values of (a) angular and (b) intensity relocation errors through time according to the GUFM1 and IGRF-9 models (dashed and continuous lines respectively). Bold (Italian maxima), dark grey (Italian latitude maxima), light grey (global maxima).

projection $(\mathrm{H})$ of the magnetic vector were selected. All the selected data (536 directions and 393 intensity values) were relocated to each one of three central locations distributed along the Italian latitude range (La Spezia, Viterbo and Naples) and their alleged relocation error was evaluated as the discrepancy between the relocated values and the ones predicted according to the GUFM1 model. The correlation between relocation distance and the mean «discrepancy» is the same found in Casas and Incoronato (2007). As an example the angular discrepancy between relocated data from the Italian catalogue and GUFM1 values at Viterbo as a function of relocation distance is plotted in fig. 3 . The correlation is apparent, $\mathrm{R}^{2}$ parameter is 0.167. To cancel out the dispersion effect the correlation parameter can be computed averaging the data at $10 \mathrm{~km}$ intervals, then $\mathrm{R}^{2}$ raises to 0.454 and using only data with a relocation distance lower than $450 \mathrm{~km}$ is already 0.815 . However some data exhibit discrepancy values that cannot be explained as solely due to relocation error.

The upper limit of discrepancy assumable as relocation error has been selected using error distributions presented in Casas and Incoronato (2007). Figure 4 shows the evolution of the maximum values of relocation errors (both angular and intensity errors) through time according to the models. Three types of maximum values have been plotted: the absolute global maxima, the maxima for the range of latitudes of Italy and finally the actual Italian maxima. The absolute global errors have kept increasing for the last 200 years, this is due to the decline of the dipole to non-dipole field ratio although it might be also partially due to the increasing resolution of the model for the most modern years. In any case, the local maximum errors (those across the Italian latitude or simply those of the Italian area) are similar and relatively constant through the time period analyzed. Therefore, the upper limits of assumable discrepancy for 

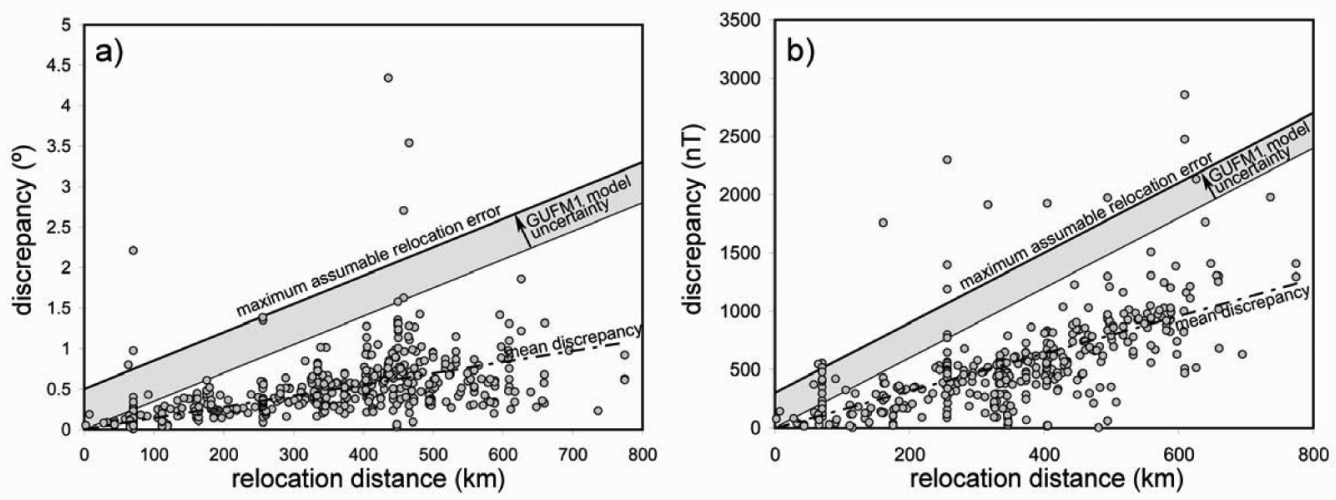

Fig. 5. Angular (a) and intensity (b) discrepancy between relocated data from the Italian catalogue and GUFM1 values at Viterbo as a function of relocation distance. Shifted line of maximum assumable relocation error has been plotted to identify anomalous data.

the Italian region have been selected as $0.35^{\circ}$ and $300 \mathrm{nT}$ per every relocated hundred of kilometres. Threshold lines have been shifted upwards $0.5^{\circ}$ and $300 \mathrm{nT}$ to account for the estimated error of GUFM1 model itself at the considered region, which is mainly due to the crustal field (Jackson, et al., 2000). This methodology allows a quick survey on long lists of geomagnetic data without the need of computing the «true» values (from a global model) at every single location but only at some relocation centres.

\subsection{Results}

Figure 5 shows the discrepancy between relocated and predicted values at Viterbo as a function of relocation distance. The shifted and unshifted upper limits have been plotted to reveal the outliers, though they show discrepancy values well above the average ones. The shift of the threshold lines reduces the number of outliers and can be considered as a cautious filter to avoid the exclusion of appropriate data. Tables I and II show the assembly of data that being relocated fail to lie below the line of assumable relocation error for at least one of the three relocation centres. These are 14 directions and 20 intensity data that could be regarded as anomalous. They only represent the $2.6 \%$ and the $5.1 \%$ of the total amount of directions and intensity values respectively.

\section{Discussion}

Although some data are revealed as erroneous for any relocation centre considered, it is noticeable that every relocation centre has a higher sensitivity to detect outliers from nearby locations. For instance, spurious data from northern Italy (Milano, Moncalieri...) are especially detected from La Spezia, whereas spurious data from Sicily (Palermo, Caltanissetta...) are particularly detected from Naples. This indicates that the defined threshold could actually be logarithmic instead of linear. However, systematic evaluations described in Casas and Incoronato (2007) point to a linear dependence of the maximum errors up to relocation distances of $1200 \mathrm{~km}$. Anyhow, this non-uniform sensitivity advises to perform this kind of analysis from a grid of relocation centres instead of a single central point.

The anomalous values could be due to locally important crustal disturbances, for instance $50 \%$ of the spurious directional data comes from Moncalieri and Lanzo Torinese (two locations from the Piedmont only $30 \mathrm{~km}$ away from each other). These data were published by a single author (Boddaert, 1907). However, most 
Table I. Directional data from the Historical Italian Geomagnetic Data Catalogue (Cafarella et al., 1992a) that fail to lie below the line of assumable relocation error for at least one of the three relocation centres: La Spezia $(\mathrm{S})$, Viterbo $(\mathrm{V})$ or Naples $(\mathrm{N})$. Failure is indicated by $\boldsymbol{\checkmark}$ sign. References are labelled according to Cafarella $e t$ al. (1992a).

\begin{tabular}{|c|c|c|c|c|c|}
\hline LOCATION & TIME & Reference & $\mathbf{S}$ & $\mathbf{V}$ & $\mathbf{N}$ \\
\hline Roma & 1640.5 & Kircher*(1643) & $\checkmark$ & $\checkmark$ & $\checkmark$ \\
\hline Napoli & 1829.5 & Fisher $^{1}$ & & & $\checkmark$ \\
\hline Roma & 1833.1 & Pianciani ${ }^{1}$ & & $\checkmark$ & \\
\hline Milano & 1863.8 & Buzzetti*(1864) & $\checkmark$ & & \\
\hline Bormio & 1887.0 & Chistoni*(1888) & $\checkmark$ & $\checkmark$ & $\checkmark$ \\
\hline Castel Piano & 1889.6 & Palazzo $^{10}$ & & $\checkmark$ & \\
\hline Castelnuovo & 1890.0 & Kesslitz $^{10}$ & $\checkmark$ & $\checkmark$ & $\checkmark$ \\
\hline Moncalieri & 1905.5 & Boddaert*(1907) & $\checkmark$ & & \\
\hline Moncalieri & 1905.5 & Boddaert*(1907) & $\checkmark$ & & \\
\hline Lanzo Torinese & 1906.0 & Boddaert*(1907) & $\checkmark$ & & \\
\hline Moncalieri & 1906.5 & Boddaert*(1907) & $\checkmark$ & & \\
\hline Moncalieri & 1906.5 & Boddaert*(1907) & $\checkmark$ & & \\
\hline Moncalieri & 1906.5 & Boddaert*(1907) & $\checkmark$ & & \\
\hline Lanzo Torinese & 1907.0 & Boddaert*(1907) & $\checkmark$ & $\checkmark$ & \\
\hline
\end{tabular}

of the data in the Italian database from these two locations and from Superga, spelled as Soperga in the database, ( 10 km away from Moncalieri) published by several authors lie just below the defined threshold. In fact, situating Moncalieri and its surrounding locations on an aeromagnetic anomaly map of Italy (Caratori-Tontini et al., 2004) one notices that they lie in a zone with important positive and negative magnetic anomalies and thus with important anomaly gradients which can result in high relocation errors. Concerning the anomalous directional data from other locations, we could presume instrumental errors for one datum from Naples (1829.5) and two data from Rome (1640.5 and 1833.1) as they are the oldest measurements (comprising both declination and inclination) from these two locations. The correlation between time and angular mismatch observed in fig. 2 (inset) supports the signs of lower quality for the oldest data. Although not being that old, possibly instrumental errors are also affecting the anomalous values registered in Bormio and Castelnuovo because they are detected for any of the three selected relocation centres and their associated relocation errors are several times the average relocation error values and thus the discrepancy cannot be merely attributed to the combined effect of relocation procedure and crustal field.

Anomalous intensity values can be partly explained by the low quality of their associated directional data which is fundamental to compute the relocation of intensity. For a significant part of the anomalous intensity data $(40 \%)$ the associated declination values were not available and thus, for the relocation of the intensity, the position of the virtual pole obtained from a 
Table II. Intensity data from the Historical Italian Geomagnetic Data Catalogue (Cafarella et al., 1992a) that fail to lie below the line of assumable relocation error for at least one of the three relocation centres: La Spezia (S), Viterbo (V) or Naples (N). Failure is indicated by $\checkmark$ sign. Values in italics indicate that the corresponding directional data (table I) also failed. References are labelled according to Cafarella et al. (1992a).

\begin{tabular}{|c|c|c|c|c|c|c|}
\hline LOCATION & TIME & Reference & $\mathbf{S}$ & $\mathbf{V}$ & $\mathbf{N}$ & \\
\hline NAPOLI & 1805.0 & Humboldt $^{33} \&$ Humboldt ${ }^{1}$ & $\checkmark$ & $\checkmark$ & $\checkmark$ & $\mathrm{a}$ \\
\hline NAPOLI & 1829.5 & Fisher $^{1}$ & & & $\checkmark$ & \\
\hline MILANO & 1834.9 & von Waltershansen ${ }^{1}$ & $\checkmark$ & $\checkmark$ & $\checkmark$ & $\mathrm{a}$ \\
\hline FIRENZE & 1835.0 & Listing $^{1}$ & $\checkmark$ & $\checkmark$ & $\checkmark$ & a \\
\hline PALERMO & 1836.0 & Listing $^{1}$ & & $\checkmark$ & $\checkmark$ & \\
\hline ROMA & 1838.4 & Bache $^{1}$ & & $\checkmark$ & & a \\
\hline MANTOVA & 1846.6 & Kreil $^{1}$ & $\checkmark$ & $\checkmark$ & $\checkmark$ & \\
\hline NAPOLI & 1859.5 & Fox $^{1}$ & & & $\checkmark$ & $\mathrm{a}$ \\
\hline RUTA & 1859.5 & Fox $^{1}$ & $\checkmark$ & & & $\mathrm{a}$ \\
\hline SPOLETO & 1859.5 & Fox $^{1}$ & & $\checkmark$ & & $\mathrm{a}$ \\
\hline NAPOLI & 1860.3 & Quetelet $^{1}$ & & $\checkmark$ & $\checkmark$ & a \\
\hline MILANO & 1863.8 & Buzzetti*(1864) & $\checkmark$ & & & \\
\hline ROMA & 1870.6 & Braun $^{1}$ & & $\checkmark$ & & \\
\hline NAPOLI & 1871.0 & Secchi ${ }^{1}$ & & & $\checkmark$ & \\
\hline TRAPANI & 1881.0 & Chistoni*(1881) & & & $\checkmark$ & \\
\hline PALERMO & 1881.9 & Chistoni*(1881) & & & $\checkmark$ & \\
\hline CATANIA & 1882.0 & Chistoni $^{10}$ & $\checkmark$ & $\checkmark$ & $\checkmark$ & \\
\hline CATANIA & 1882.0 & Chistoni $^{10}$ & $\checkmark$ & $\checkmark$ & $\checkmark$ & \\
\hline ARENZANO & 1885.9 & Chistoni $^{10}$ & $\checkmark$ & & & \\
\hline CALTANISETTA & 1890.6 & Chistoni $^{10}$ & & & $\checkmark$ & \\
\hline
\end{tabular}

${ }^{\mathrm{a}}$ Declination values were not available.

nearby location datum of similar age was used. The higher incidence of such kind of values compared with their occurrence $(9 \%)$ within the general database of intensity values is evidence for the additional source of error due to the lack of knowledge of declination. Two more data have directional values already detected as anomalous and thus the corresponding relocated intensity could lack of meaning. It is worth noting that in these cases the original (non-relocated) intensity values could be in fact not erroneous at all. Finally, from the 9 remaining anomalous intensity values it is remarkable the high rate of data from Sicily (6 values from 5 
different locations) although it is difficult to explain this geographical concentration in terms of crustal anomalies as the Sicilian directional data does not appear as doubtful.

\section{Conclusions}

The distribution of relocation errors calculated using IGRF-9 and GUFM1 models has been applied to a historical geomagnetic database (the Historical Italian Geomagnetic Data Catalogue) to detect abnormal data. Specific conclusions (on the Italian database) and general conclusions (on the approach used) can be drawn.

The Italian database has proven to contain reliable data, only a very low percentage of data (14 directions and 20 intensities) can be considered anomalous. This catalogue could thus be added to the databases used to produce regional or global geomagnetic models. Some reasons can be hypothesized to explain some of the outliers: i) poor quality of the measurements, this appears to correlate with the oldest data, ii) intensity data can appear anomalous as a consequence of the poor quality of their associated directional data, iii) local crustal disturbances of the internal field, grounds for this effect are found for several data from the Piedmont area.

The latter reason implies that not all anomalous values are actually erroneous; discrepancies can arise from the fact that GUFM1 model was devised as a model for the core-mantle boundary and its extrapolation to the Earth's surface disregards crustal contributors.

The described methodology takes advantage of the higher resolution of the geomagnetic models that describe the present field to define the limits to decide whether a value is consistent or requires additional explanation. Simple comparison between the historical geomagnetic database values and its corresponding values computed from a model would evade an overview on the global structure of the field and it would be difficult to establish acceptance limits as there are no uncertainty indications related to the values predicted by the model.

A secondary advantage of the described methodology over the simple comparison between every single data and its value computed from a model is precisely the fact that it is not necessary to compute all the data through the model. This could be important whenever the model is difficult to compute or simply not computable for some areas (e.g. when using a non-global model to evaluate data that lie outside the application limits of the model, the data could be relocated to a centre within the application area). Finally, even without any model at all, the approach presented here could be used as a way to check the self-consistency of any assembly of data by relocating contemporaneous data to every neighbouring available data location. This should be helpful in areas where the existing models predict the field based on only a few historical records and specially when dealing with assemblies of archaeomagnetic data from a given area.

\section{Acknowledgments}

Financial support from AARCH - Research Training Network (UE, contract n. PRNCT2002-00219) and EME2005-42 projects is acknowledged. The paper benefited from constructive reviews by two anonymous reviewers.

\section{REFERENCES}

BOODAERT, D. (1907): Misure magnetiche nei dintorni di Torino. Declinazione e inclinazione, Memoria della $R$. Accademia delle Scienze di Torino, 58, series 2, 397-450.

Cafarella, L., A. De Santis and A. Meloni (1992a): The Historical Italian Geomagnetic Data Catalogue, ING, Rome, pp. 160.

Cafarella, L., A. De Santis and A. Meloni (1992b): Secular variation in Italy from historical geomagnetic field measurements, Phys. of the Earth and Planet. Int., 73 (3-4), 206-221.

Caratori-Tontini, F., P. Stefanelli, I. Giori, O. Faggioni and C. CARMISCIANO (2004): The revised aeromagnetic anomaly map of Italy, Annals of Geophysics, 47 (5), $1547-1555$

CASAS, LL. and A. InCORONATO, (2007): Distribution analysis of errors due to relocation of geomagnetic data using the «Conversion via Pole» (CVP) method: implications on archaeomagnetic data, Geophys. J. Int., 169, 448-454.

JACKSON, A., A Jonkers, and M. WALKER (2000): Four centuries of geomagnetic secular variation from historical records, Phil. Trans. R. Soc. Lond., A. 358, 957-990.

Korte, M. and C.G. Constable, (2005): The geomagnetic 
dipole moment over the last 7000 years-new results from a global model, Earth and Planet. Sc. Letters, 236, 348-358.

Maus, S., S. Macmillan, T. Chernova, S. Choi, D. Dater, V. Golovkov, V. Lesur, F. Lowes, H. LÜHr, W. MaI, S. Mclean, N. Olsen, M. Rother, T. Sabaka, A.
Thomson and T. Zvereva (2005): The 10th Generation International Geomagnetic Reference Field, Phys. of the Earth and Planet. Int. 151 (3-4), 320-322.

(received March 26, 2008; accepted April 23, 2008) 